\title{
Performance of Mulches and Micronutrients on Water Use of Broccoli (Brassica oleracea L var. Italica Plenck)
}

\author{
B.V.G. Prasad* and S. Chakravorty \\ Department of Horticulture \& PHT, Institute of Agriculture, \\ Visva-Bharati University, Sriniketan, West-Bengal, India-731236 \\ *Corresponding author
}

A B S T R A C T

\section{Keywords}

Broccoli,

Micronutrients,

Mulches, Water use and yield

Article Info

Accepted:

04 December 2018

Available Online:

10 January 2019

\begin{abstract}
The Field experiment was conducted during rabi season of 2014-15 and 2015-16 to find out the response of mulches and micronutrients on growth and yield of broccoli with an objective to estimate water use and water use efficiency of broccoli. The experiment was laid out in split-plot design with three main plots (mulches), nine sub plots (micronutrients) and twenty seven interaction treatments (mulches $\mathrm{x}$ micro nutrients) and each treatment was replicated thrice. The results revealed that mulches and micronutrients as well as their interaction significantly affected the water use and water use efficiency of broccoli. The lowest water use $(153.86 \mathrm{~mm})$ and highest water use efficiency $(76.36 \mathrm{~kg}$ ha $\mathrm{mm})$ were observed were with application of paddy straw mulch-7t.ha ${ }^{-1}$ along with combined application of $\mathrm{Zn}-1 \%+\mathrm{B}-0.5 \%$ in broccoli.
\end{abstract}

\section{Introduction}

Broccoli (Brassica oleracea L. var. Italica) is one of the most nutritious of cole crops which contains high amount of Vitamin-A (9000IU/100g), protein $(3.5 \%)$ (Friedman, 1996), antioxidants, phenolics, vitamins and other bioactive compounds (viz., sulphoraphane, total dietary fiber, minerals, folates, etc.). Plant nutrition is one of the prime considerations for getting higher yield (Saha et al., 2010) and it was observed that balanced application of fertilizers is the prerequisite for obtaining higher yield and better quality of broccoli (Brahma et al.,
2002). Micronutrients are essential for plant growth and play an important role in balanced crop nutrition. Among micronutrients, zinc and boron are important for broccoli production. Zinc is a precursor of auxins and it helps formation of chlorophyll in leaves thereby increases photosynthesis for better growth and development of the plant (Torres, 1974). Deficiency of zinc led to development of small sized heads with poor market price. Boron is responsible for increased translocation of photosynthates from source (leaves) to sink (head) (Pandhawa and Bhail, 1976) and thus helps to attain high head weight thereby high yield. The deficiency of 
boron led to browning and hollow heart of broccoli with poor physical appearance and low consumer acceptance in the market. Water management is one of the important factors to obtain higher yield. Demand of water and nutrients was higher at the time of flowering and decreased thereafter Vitkov (1975). The lateritic belt of West Bengal, water stress is common phenomenon due to acute soil moisture deficit, deep ground water table and inadequate distribution of rainfall. Therefore, adoption of cheap and easily applicable water conservation techniques is necessary for obtaining high yields. Among the different techniques used for water conservation, mulching has been proved to be the best for its simplicity and cost-effectiveness. Estimation of water use and water use efficiency under specific type of mulches help develop an idea about optimum water requirement. Hence, the present study was undertaken to evaluate the effect of mulching on water use and water use efficiency of broccoli.

\section{Materials and Methods}

The field experiment was conducted at the Horticultural Farm, Institute of Agriculture, Visva-Bharati University during rabi season of 2014-15 and 2015-16. The experimental field was situated in semi-arid, sub-humid zone of West Bengal ( $23^{\circ} 42^{\prime} \mathrm{N}$ latitude and $87^{\circ} 40^{\prime} 30^{\prime \prime} \mathrm{E}$ longitude), India. The soil of the experiment was sandy loam with $\mathrm{pH}$ of 5.86.1 having low organic matter $(0.63 \%)$. The experiment was laid out in split plot design with three main plots (mulches), nine sub plots (micronutrients) and twenty seven interaction (mulches $\mathrm{x}$ micro nutrients) treatments. According to the experimental design plots were prepared by ploughing followed by harrowing. Well prepared plots were manured with FYM @20 t.ha ${ }^{-1}$ and fertilized with recommended dose of N: P: K @ 100:80:100 $\mathrm{kg} / \mathrm{ha}$, respectively, in the form of urea, super phosphate (SSP), muriate of potash (MOP).
The full dose of $\mathrm{P}$ and $\mathrm{K}$ and half of $\mathrm{N}$ was applied as basal and the remaining amount of $\mathrm{N}$ was top-dressed 30 days after transplanting (DAT). Seedlings were transplanted at $60 \mathrm{~cm} \mathrm{x}$ $40 \mathrm{~cm}$ spacing and mulches were applied uniformly after establishment of seedlings. Foliar application of micro nutrients carried at 30 and 45 DAT and measured quantity of life saving irrigation was applied to each plot uniformly. Total number of six irrigations was adjusted along with rainfalls.

In each irrigation measured quantity of water i.e. 100 and 100 liters in first and second, 150 and 150 liters in third and fourth, 200 and 200 liters were applied at fifth and sixth irrigation to the crop and other recommended package of practices was followed to raise a good crop. Water use (mm) was calculated based on applied irrigation water + effective rain fall during crop growth + soil moisture content of the treatment. Regarding WUE, it was calculated based on effect of water use on pod yield \& expressed $\mathrm{kg}$ ha $\mathrm{mm}$. Data on available soil nutrient status was worked out after completion of the experiment. Data were statistically processed by the analysis of variance method (Panse and Sukhatme, 1978) $\&$ the significance of variance was tested.

\section{Results and Discussion}

Applied mulches, micronutrients and their interactions significantly influenced the water use in broccoli and increased water use was observed in the second year of the crop growth over the first year. It was manifested that mulching with paddy straw mulch @7 t.ha ${ }^{-1}$ $\left(\mathrm{M}_{1}\right)$ remarkably reduced the water use to $201.46 \mathrm{~mm}$ over application of water hyacinth mulch of same quantity and non-application. It was also observed that application of paddy straw mulch@7 t ha ${ }^{-1}$ saved water up to 75.34 $\mathrm{mm}$ and reduced the requirement to $37.39 \%$ over non-application of mulches $\left(\mathrm{M}_{3}\right)$. 
Table.1 Effect of mulches and micro nutrients on water use (mm) of broccoli

\begin{tabular}{|c|c|c|c|}
\hline Mulches (M) & 2014-2015 & 2015-2016 & Pooled \\
\hline M1.Paddy straw mulch@7t/ha & 200.75 & 202.16 & 201.46 \\
\hline 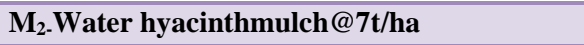 & 221.56 & 222.82 & 222.19 \\
\hline $\mathrm{M}_{3-\text { No mulch }}$ & 276.29 & 277.31 & 276.80 \\
\hline S. Em \pm & 0.61 & 0.58 & 0.58 \\
\hline CD (0.05) & 2.39 & 2.26 & 2.29 \\
\hline \multicolumn{4}{|l|}{ Micro nutrients (mn) } \\
\hline $\mathrm{mn}_{1} \cdot \mathbf{Z n}_{0}(\mathbf{0 \%})+\mathrm{Bo}_{0}(\mathbf{0 \%})$ & 237.52 & 238.49 & 238.01 \\
\hline $\mathrm{mn}_{2} . \mathrm{Zn}_{1}(0.5 \%)+\mathrm{Bo}_{0}(0 \%)$ & 234.01 & 235.10 & 234.55 \\
\hline $\mathrm{mn}_{3} \cdot \mathrm{Zn}_{2}(1 \%)+\mathrm{Bo}_{0}(0 \%)$ & 234.45 & 236.60 & 235.52 \\
\hline $\mathrm{mn}_{4} \cdot \mathrm{Zn}_{0}(0 \%)+\mathrm{Bo}_{1}(0.5 \%)$ & 236.32 & 237.21 & 236.77 \\
\hline $\mathrm{mn}_{5} . \mathrm{Zn}_{1}(0.5 \%)+\mathrm{Bo}_{1}(0.5 \%)$ & 232.07 & 232.92 & 232.50 \\
\hline $\mathrm{mn}_{6} \cdot \mathrm{Zn}_{2}(1 \%)+\mathrm{Bo}_{1}(0.5 \%)$ & 227.69 & 229.37 & 228.53 \\
\hline $\mathrm{mn}_{7} \cdot \mathrm{Zn}_{0}(0 \%)+\mathrm{Bo}_{2}(1 \%)$ & 233.14 & 234.21 & 233.68 \\
\hline $\mathrm{mn}_{8} \cdot \mathrm{Zn}_{1}(0.5 \%)+\mathrm{Bo}_{2}(1 \%)$ & 229.57 & 230.88 & 230.22 \\
\hline $\mathrm{mn}_{9} . \mathrm{Zn}_{2}(1 \%)+\mathrm{Bo}_{2}(1 \%)$ & 231.04 & 232.12 & 231.58 \\
\hline S.Em \pm & 1.31 & 1.26 & 1.27 \\
\hline CD (0.05) & 3.73 & 3.58 & 3.62 \\
\hline \multicolumn{4}{|l|}{ Interaction (M x mn) } \\
\hline $\mathrm{M}_{1} \mathrm{mn}_{1}-\mathrm{PM}-7 \mathrm{t} / \mathrm{ha}+\mathrm{Zn}_{0}(0 \%)+\mathrm{Bo} \mathrm{o}_{0}(0 \%)$ & 205.93 & 207.29 & 206.61 \\
\hline $\mathrm{M}_{1} \mathrm{mn}_{2}-\mathrm{PM}-7 \mathrm{t} / \mathrm{ha}+\mathrm{Zn}_{1}(0.5 \%)+\mathrm{Bo}_{0}(0 \%)$ & 202.37 & 203.50 & 202.94 \\
\hline $\mathrm{M}_{1} \mathrm{mn}_{3}-\mathrm{PM}-7 \mathrm{t} / \mathrm{ha}+\mathrm{Zn}_{2}(1 \%)+\mathrm{Bo}_{0}(0 \%)$ & 202.71 & 206.00 & 204.35 \\
\hline $\mathrm{M}_{1} \mathrm{mn}_{4}-\mathrm{PM}-7 \mathrm{t} / \mathrm{ha}+\mathrm{Zn}_{0}(0 \%)+\mathrm{Bo}_{1}(0.5 \%)$ & 204.62 & 204.82 & 204.72 \\
\hline $\mathrm{M}_{1} \mathrm{mn}_{5}-\mathrm{PM}-7 \mathrm{t} / \mathrm{ha}+\mathrm{Zn}_{1}(0.5 \%)+\mathrm{Bo}_{1}(0.5 \%)$ & 199.67 & 200.79 & 200.23 \\
\hline $\mathrm{M}_{1} \mathrm{mn}_{6}-\mathrm{PM}-7 \mathrm{t} / \mathrm{ha}+\mathrm{Zn}_{2}(1 \%)+\mathrm{Bo}_{1}(0.5 \%)$ & 194.72 & 196.29 & 195.50 \\
\hline $\mathrm{M}_{1} \mathrm{mn}_{7}-\mathrm{PM}-7 \mathrm{t} / \mathrm{ha}+\mathrm{Zn}_{0}(0 \%)+\mathrm{Bo}_{2}(1 \%)$ & 201.34 & 202.45 & 201.90 \\
\hline $\mathrm{M}_{1} \mathrm{mn}_{8}-\mathrm{PM}-7 \mathrm{t} / \mathrm{ha}+\mathrm{Zn}_{1}(0.5 \%)+\mathrm{Bo}_{2}(1 \%)$ & 196.60 & 198.23 & 197.42 \\
\hline $\mathrm{M}_{1} \mathrm{mn}_{9}-\mathrm{PM}-7 \mathrm{t} / \mathrm{ha}+\mathrm{Zn}_{2}(1 \%)+\mathrm{Bo}_{2}(1 \%)$ & 198.81 & 200.11 & 199.46 \\
\hline $\mathrm{M}_{2} \mathrm{mn}_{1}-\mathrm{WHM}-7 \mathrm{t} / \mathrm{ha}+\mathrm{Zn}_{0}(0 \%)+\mathrm{Bo}_{0}(0 \%)$ & 225.04 & 225.84 & 225.44 \\
\hline $\mathrm{M}_{2} \mathrm{mn}_{2}-\mathrm{WHM}-7 \mathrm{t} / \mathrm{ha}+\mathrm{Zn}_{1}(0.5 \%)+\mathrm{Bo}_{0}(0 \%)$ & 222.31 & 223.40 & 222.86 \\
\hline $\mathrm{M}_{2} \mathrm{mn}_{3}-\mathrm{WHM}-7 \mathrm{t} / \mathrm{ha}+\mathrm{Zn}_{2}(1 \%)+\mathrm{Bo}_{0}(0 \%)$ & 222.67 & 225.01 & 223.84 \\
\hline $\mathrm{M}_{2} \mathrm{mn}_{4}-\mathrm{WHM}-7 \mathrm{t} / \mathrm{ha}+\mathrm{Zn}_{0}(0 \%)+\mathrm{Bo}_{1}(0.5 \%)$ & 224.87 & 226.13 & 225.50 \\
\hline $\left.\mathrm{M}_{2} \mathrm{mn}_{5}-\mathrm{WHM}-7 \mathrm{t} / \mathrm{ha}+\mathrm{Zn}_{1}(0.5 \%)+\mathrm{Bo}_{1} 0.5 \%\right)$ & 221.50 & 221.70 & 221.60 \\
\hline $\mathrm{M}_{2} \mathrm{mn}_{6}-\mathrm{WHM}-7 \mathrm{t} / \mathrm{ha}+\mathrm{Zn}_{2}(1 \%)+\mathrm{Bo}_{1}(0.5 \%)$ & 217.43 & 219.53 & 218.48 \\
\hline $\mathrm{M}_{2} \mathrm{mn}_{7}-\mathrm{WHM}-7 \mathrm{t} / \mathrm{ha}+\mathrm{Zn}_{0}(0 \%)+\mathrm{Bo}_{2}(1 \%)$ & 221.49 & 222.40 & 221.94 \\
\hline $\mathrm{M}_{2} \mathrm{mn}_{8}-\mathrm{WHM}-7 \mathrm{t} / \mathrm{ha}+\mathrm{Zn}_{1}(0.5 \%)+\mathrm{Bo}_{2}(1 \%)$ & 218.60 & 219.79 & 219.19 \\
\hline $\mathrm{M}_{2} \mathrm{mn}_{9}-\mathrm{WHM}-7 \mathrm{t} / \mathrm{ha}+\mathrm{Zn}_{2}(1 \%)+\mathrm{Bo}_{2}(1 \%)$ & 220.15 & 221.61 & 220.88 \\
\hline $\mathrm{M}_{3} \mathrm{mn}_{1}-\mathrm{NM}+\mathrm{Zn}_{0}(0 \%)+\mathrm{Bo} \mathrm{o}_{0}(0 \%)$ & 281.60 & 282.34 & 281.97 \\
\hline $\mathrm{M}_{3} \mathrm{mn}_{2}-\mathrm{NM}+\mathrm{Zn}_{1}(0.5 \%)+\mathrm{Bo}_{0}(0 \%)$ & 277.34 & 278.40 & 277.87 \\
\hline $\mathrm{M}_{3} \mathrm{mn}_{3}-\mathrm{NM}+\mathrm{Zn}_{2}(1 \%)+\mathrm{Bo}_{0}(0 \%)$ & 277.97 & 278.79 & 278.38 \\
\hline $\mathrm{M}_{3} \mathrm{mn}_{4}-\mathrm{NM}+\mathrm{Zn}_{0}(0 \%)+\mathrm{Bo}_{1}(0.5 \%)$ & 279.48 & 280.69 & 280.08 \\
\hline $\mathrm{M}_{3} \mathrm{mn}_{5}-\mathrm{NM}+\mathrm{Zn}_{1}(0.5 \%)+\mathrm{Bo}_{1}(0.5 \%)$ & 275.04 & 276.29 & 275.66 \\
\hline $\mathrm{M}_{3} \mathrm{mn}_{6}-\mathrm{NM}+\mathrm{Zn}_{2}(1 \%)+\mathrm{Bo}_{1}(0.5 \%)$ & 270.93 & 272.29 & 271.61 \\
\hline $\mathrm{M}_{3} \mathrm{mn}_{7}-\mathrm{NM}+\mathrm{Zn}_{0}(0 \%)+\mathrm{Bo}_{2}(1 \%)$ & 276.60 & 277.79 & 277.19 \\
\hline $\mathrm{M}_{3} \mathrm{mn}_{8}-\mathrm{NM}+\mathrm{Zn} \mathrm{n}_{1}(0.5 \%)+\mathrm{Bo}_{2}(1 \%)$ & 273.50 & 274.63 & 274.07 \\
\hline $\mathrm{M}_{3} \mathrm{mn}_{9}-\mathrm{NM}+\mathrm{Zn}_{2}(1 \%)+\mathrm{Bo}_{2}(1 \%)$ & 274.17 & 274.64 & 274.40 \\
\hline S.Em \pm & 2.27 & 2.18 & 2.20 \\
\hline CD (0.05) & 6.45 & 6.20 & 6.27 \\
\hline
\end{tabular}


Table.2 Effect of mulches and micro nutrients on water use efficiency (Kg/ha/mm) of broccoli

\begin{tabular}{|c|c|c|c|}
\hline Mulches (M) & 2014-2015 & 2015-2016 & Pooled \\
\hline M1. Paddy straw mulch@7t/ha & 75.08 & 74.92 & 75.00 \\
\hline M2-Water hyacinthmulch@7t/ha & 61.99 & 63.27 & 62.63 \\
\hline $\mathbf{M}_{3-}$ No mulch & 45.57 & 47.28 & 46.43 \\
\hline S. Em \pm & 0.80 & 0.74 & 0.47 \\
\hline CD (0.05) & 3.16 & 2.90 & 1.83 \\
\hline \multicolumn{4}{|l|}{ Micro nutrients (mn) } \\
\hline $\mathrm{mn}_{1} . \mathrm{Zn}_{0}(\mathbf{0 \%})+\mathrm{Bo}_{0}(\mathbf{0 \%})$ & 48.31 & 49.51 & 48.91 \\
\hline $\mathrm{mn}_{2} . \mathrm{Zn}_{1}(0.5 \%)+\mathrm{Bo} 0(0 \%)$ & 58.19 & 61.28 & 59.74 \\
\hline $\mathrm{mn}_{3} \cdot \mathbf{Z n}_{2}(1 \%)+\mathbf{B o}_{0}(0 \%)$ & 57.51 & 60.82 & 59.16 \\
\hline $\mathrm{mn}_{4} \cdot \mathrm{Zn}_{0}(0 \%)+\mathrm{Bo}_{1}(0.5 \%)$ & 55.35 & 54.52 & 54.93 \\
\hline $\mathrm{mn}_{5} . \mathrm{Zn}_{1}(0.5 \%)+\mathrm{Bo}_{1}(0.5 \%)$ & 64.77 & 61.71 & 63.24 \\
\hline $\mathrm{mn}_{6} \cdot \mathrm{Zn}_{2}(1 \%)+\mathrm{Bo}_{1}(0.5 \%)$ & 70.78 & 72.30 & 71.54 \\
\hline $\mathrm{mn}_{7} . \mathrm{Zn}_{0}(0 \%)+\mathrm{Bo}_{2}(1 \%)$ & 59.91 & 61.43 & 60.67 \\
\hline $\mathrm{mn}_{8} \cdot \mathrm{Zn}_{1}(0.5 \%)+\mathrm{Bo}_{2}(1 \%)$ & 67.56 & 68.93 & 68.25 \\
\hline $\mathrm{mn}_{9} . \mathrm{Zn}_{2}(1 \%)+\mathrm{Bo}_{2}(1 \%)$ & 65.57 & 65.93 & 65.75 \\
\hline S.Em \pm & 1.22 & 1.29 & 0.93 \\
\hline CD (0.05) & 3.47 & 3.67 & 2.64 \\
\hline \multicolumn{4}{|l|}{ Interaction (M x mn) } \\
\hline $\mathrm{M}_{1} \mathrm{mn}_{1}-\mathrm{PM}-7 \mathrm{t} / \mathrm{ha}+\mathrm{Zn}_{0}(0 \%)+\mathrm{Bo}_{0}(0 \%)$ & 54.64 & 58.53 & 56.59 \\
\hline $\mathrm{M}_{1} \mathrm{mn}_{2}-\mathrm{PM}-7 \mathrm{t} / \mathrm{ha}+\mathrm{Zn}_{1}(0.5 \%)+\mathrm{Bo}_{0}(0 \%)$ & 67.57 & 68.14 & 67.85 \\
\hline $\mathrm{M}_{1} \mathrm{mn}_{3}-\mathrm{PM}-7 \mathrm{t} / \mathrm{ha}+\mathrm{Zn}_{2}(1 \%)+\mathrm{Bo}_{0}(0 \%)$ & 67.16 & 73.90 & 70.54 \\
\hline $\mathrm{M}_{1} \mathrm{mn}_{4}-\mathrm{PM}-7 \mathrm{t} / \mathrm{ha}+\mathrm{Zn}_{0}(0 \%)+\mathrm{Bo}_{1}(0.5 \%)$ & 71.96 & 69.54 & 70.75 \\
\hline $\mathrm{M}_{1} \mathrm{mn}_{5}-\mathrm{PM}-7 \mathrm{t} / \mathrm{ha}+\mathrm{Zn}_{1}(0.5 \%)+\mathrm{Bo}_{1}(0.5 \%)$ & 82.81 & 74.68 & 78.73 \\
\hline $\mathrm{M}_{1} \mathrm{mn}_{6}-\mathrm{PM}-7 \mathrm{t} / \mathrm{ha}+\mathrm{Zn}_{2}(1 \%)+\mathrm{Bo}_{1}(0.5 \%)$ & 87.81 & 89.26 & 88.54 \\
\hline $\mathrm{M}_{1} \mathrm{mn}_{7}-\mathrm{PM}-7 \mathrm{t} / \mathrm{ha}+\mathrm{Zn}_{0}(0 \%)+\mathrm{Bo}_{2}(1 \%)$ & 73.72 & 73.50 & 73.61 \\
\hline $\mathrm{M}_{1} \mathrm{mn}_{8}-\mathrm{PM}-7 \mathrm{t} / \mathrm{ha}+\mathrm{Zn}_{1}(\mathbf{0 . 5 \%})+\mathrm{Bo}_{2}(1 \%)$ & 85.18 & 85.11 & 85.15 \\
\hline $\mathrm{M}_{1} \mathrm{mn}_{9}-\mathrm{PM}-7 \mathrm{t} / \mathrm{ha}+\mathrm{Zn}_{2}(1 \%)+\mathrm{Bo}_{2}(1 \%)$ & 84.91 & 81.65 & 83.27 \\
\hline $\mathrm{M}_{2} \mathrm{mn}_{1}-\mathrm{WHM}-7 \mathrm{t} / \mathrm{ha}+\mathrm{Zn}_{0}(0 \%)+\mathrm{Bo}(0 \%)$ & 51.06 & 52.99 & 52.03 \\
\hline $\mathrm{M}_{2} \mathrm{mn}_{2}-\mathrm{WHM}-7 \mathrm{t} / \mathrm{ha}+\mathrm{Zn}_{1}(0.5 \%)+\mathrm{Bo}_{0}(0 \%)$ & 64.26 & 67.01 & 65.64 \\
\hline $\mathrm{M}_{2} \mathrm{mn}_{3}-\mathrm{WHM}-7 \mathrm{t} / \mathrm{ha}+\mathrm{Zn}_{2}(1 \%)+\mathrm{Bo}_{0}(0 \%)$ & 60.81 & 60.93 & 60.85 \\
\hline $\mathrm{M}_{2} \mathrm{mn}_{4}-\mathrm{WHM}-7 \mathrm{t} / \mathrm{ha}+\mathrm{Zn}_{0}(0 \%)+\mathrm{Bo}_{1}(0.5 \%)$ & 50.60 & 51.74 & 51.17 \\
\hline $\left.\mathrm{M}_{2} \mathrm{mn}_{5}-\mathrm{WHM}-7 \mathrm{t} / \mathrm{ha}+\mathrm{Zn}_{1}(0.5 \%)+\mathrm{Bo}_{1} 0.5 \%\right)$ & 66.50 & 64.02 & 65.26 \\
\hline $\mathrm{M}_{2} \mathrm{mn}_{6}-W H M-7 \mathrm{t} / \mathrm{ha}+\mathrm{Zn}_{2}(1 \%)+\mathrm{Bo}_{1}(0.5 \%)$ & 73.30 & 73.03 & 73.16 \\
\hline $\mathrm{M}_{2} \mathrm{mn}_{7}-\mathrm{WHM}-7 \mathrm{t} / \mathrm{ha}+\mathrm{Zn}_{0}(0 \%)+\mathrm{Bo}_{2}(1 \%)$ & 58.64 & 61.66 & 60.15 \\
\hline $\mathrm{M}_{2} \mathrm{mn}_{8}-\mathrm{WHM}-7 \mathrm{t} / \mathrm{ha}+\mathrm{Zn}_{1}(0.5 \%)+\mathrm{Bo}_{2}(1 \%)$ & 68.81 & 70.23 & 69.53 \\
\hline $\mathrm{M}_{2} \mathrm{mn}_{9}-\mathrm{WHM}-7 \mathrm{t} / \mathrm{ha}+\mathrm{Zn}_{2}(1 \%)+\mathrm{Bo}_{2}(1 \%)$ & 63.97 & 67.84 & 65.91 \\
\hline $\mathrm{M}_{3} \mathrm{mn}_{1}-\mathrm{NM}+\mathrm{Zn}_{0}(0 \%)+\mathrm{Bo}_{0}(0 \%)$ & 39.23 & 37.01 & 38.12 \\
\hline $\mathrm{M}_{3} \mathrm{mn}_{2}-\mathrm{NM}+\mathrm{Zn}_{1}(0.5 \%)+\mathrm{Bo}_{0}(0 \%)$ & 42.74 & 48.69 & 45.72 \\
\hline $\mathrm{M}_{3} \mathrm{mn}_{3}-\mathrm{NM}+\mathrm{Zn}_{2}(1 \%)+\mathrm{Bo}_{0}(0 \%)$ & 44.55 & 47.62 & 46.09 \\
\hline $\mathrm{M}_{3} \mathrm{mn}_{4}-\mathrm{NM}+\mathrm{Zn}_{0}(0 \%)+\mathrm{Bo}_{1}(0.5 \%)$ & 43.49 & 42.28 & 42.88 \\
\hline $\mathrm{M}_{3} \mathrm{mn}_{5}-\mathrm{NM}+\mathrm{Zn}_{1}(0.5 \%)+\mathrm{Bo}_{1}(0.5 \%)$ & 45.00 & 46.43 & 45.71 \\
\hline $\mathrm{M}_{3} \mathrm{mn}_{6}-\mathrm{NM}+\mathrm{Zn}_{2}(1 \%)+\mathrm{Bo}_{1}(0.5 \%)$ & 51.24 & 54.59 & 52.92 \\
\hline $\mathrm{M}_{3} \mathrm{mn}_{7}-\mathrm{NM}+\mathrm{Zn}_{0}(0 \%)+\mathrm{Bo}_{2}(1 \%)$ & 47.36 & 49.12 & 48.24 \\
\hline $\mathrm{M}_{3} \mathrm{mn}_{8}-\mathrm{NM}+\mathrm{Zn} \mathrm{n}_{1}(0.5 \%)+\mathrm{Bo}_{2}(1 \%)$ & 48.69 & 51.44 & 50.07 \\
\hline $\mathrm{M}_{3} \mathrm{mn}_{9}-\mathrm{NM}+\mathrm{Zn}_{2}(\mathbf{1 \%})+\mathrm{Bo}_{2}(1 \%)$ & 47.83 & 48.31 & 48.07 \\
\hline S.Em \pm & 2.11 & 2.24 & 1.61 \\
\hline CD (0.05) & 6.00 & 6.36 & 4.57 \\
\hline
\end{tabular}


Fig.1 Effect of mulches and micro nutrients on water use ( $\mathrm{mm}$ ) and water use efficiency $(\mathrm{Kg} / \mathrm{ha} / \mathrm{mm})$ of broccoli

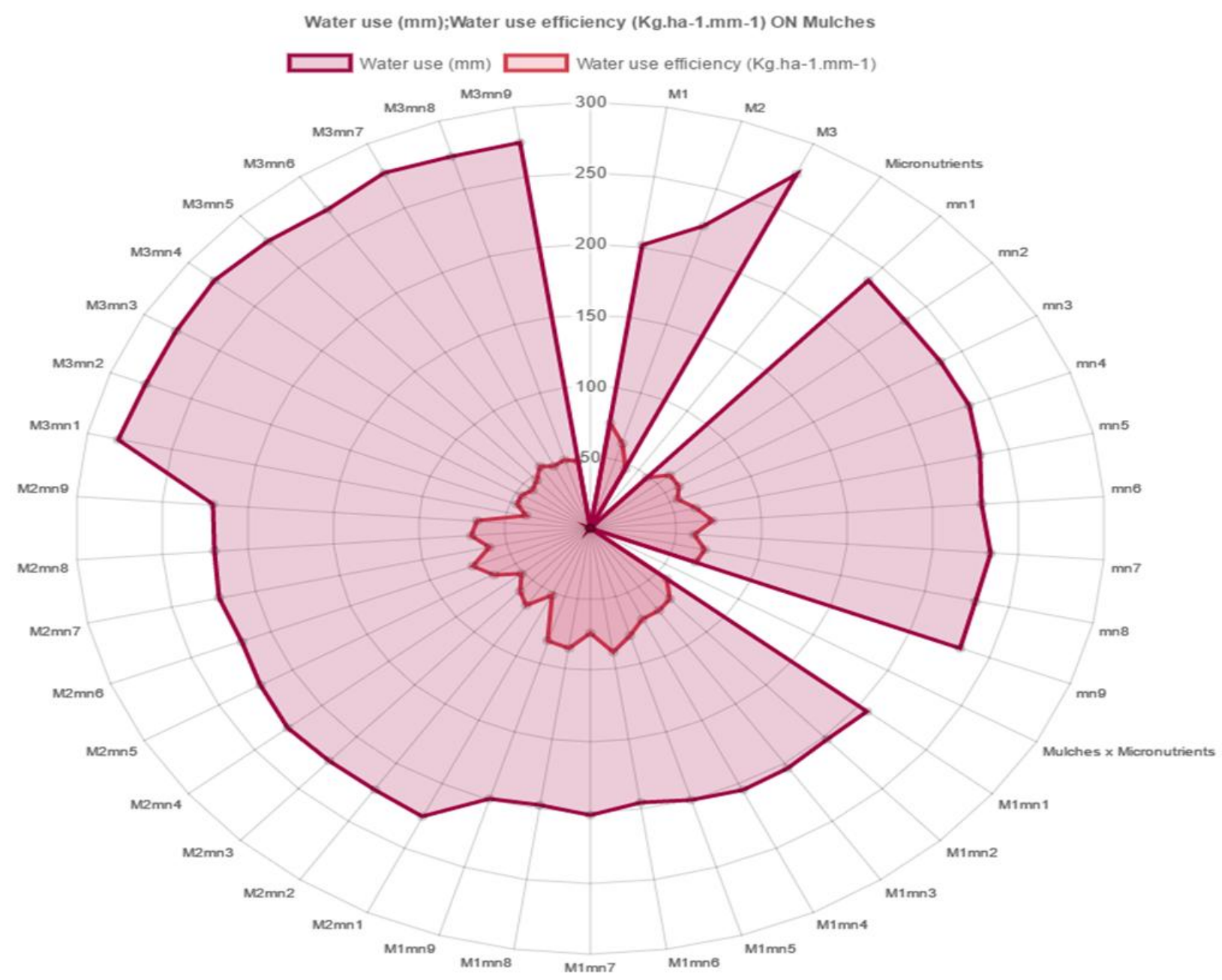

On the other hand, treatment with highest level of zinc (1\%) along with subsequent level of boron $(0.5 \%)$ (i.e. $\left.\mathrm{mn}_{6}\right)$ remarkably reduce the water use to $228.53 \mathrm{~mm}$ and it was closely followed by $\mathrm{mn}_{8}(230.22 \mathrm{~mm})$ and $\mathrm{mn}_{9}$ (231.58mm). Interaction between mulches and micronutrients (Table 1 and Fig. 1) after pooled analysis indicated their significant influence on water use. Remarkably lowest water use $(195.50 \mathrm{~mm})$ was observed where plants grown under paddy straw much along with foliar nutrition of $\mathrm{Zn}_{2}-1 \%+\mathrm{Bo}_{1}-0.5 \%$ $\left(\mathrm{M}_{1} \mathrm{mn}_{6}\right)$. Application of paddy straw mulch@ 7t.ha ${ }^{-1}$ remarkably increased the water use efficiency up to $75.00 \quad \mathrm{Kg} \cdot \mathrm{ha}^{-1}$. $\mathrm{mm}^{-1}$. On the other hand, prominent effect of applied micronutrients (i.e. $\mathrm{mn}_{6} \cdot \mathrm{Zn}_{2}-1 \%+\mathrm{Bo}_{1^{-}}$ $0.5 \%$ ) exerted maximum water use efficiency (71.54 Kg.ha ${ }^{-1} \cdot \mathrm{mm}^{-1}$ ) over other micronutrients applied to the crop. Results reviled for $\mathrm{mn}_{8}\left(68.25 \mathrm{Kg} \cdot \mathrm{ha}^{-1} \cdot \mathrm{mm}^{-1}\right)$ and $\mathrm{mn}_{9}$ $\left(65.75 \mathrm{Kg} \cdot \mathrm{ha}^{-1} \cdot \mathrm{mm}^{-1}\right)$ were at par with each other. Combined application of mulches and micronutrients significantly increased the water use efficiency over sole application and the range observed from 38.12-88.54 Kg.ha ${ }^{-1}$. $\mathrm{mm}^{-1}$. The highest water use efficiency $(88.54$ $\mathrm{Kg} \cdot \mathrm{ha}^{-1} \cdot \mathrm{mm}^{-1}$ ) was observed in plants grown 
under paddy straw mulch @ 7 t.ha ${ }^{-1}$ and treated with $\mathrm{Zn}_{2}-1 \%+\mathrm{Bo}_{1}-0.5 \% \quad\left(\mathrm{M}_{1} \mathrm{mn}_{6}\right)$, though, it was at par with $\mathrm{M}_{1} \mathrm{mn}_{8}$ (85.15 Kg.ha ${ }^{-1} \cdot \mathrm{mm}^{-1}$ ) (Table 2 and Fig. 1).

Water use and water use efficiency are interrelated terms. The lowest water use and highest water use efficiency of paddy straw might be due to its thick carpet like cover over the soil surface which reduced evaporation of water from the soil. Moreover, numerous reports indicating that straw mulching favorably influences the soil moisture regime by reducing evaporation from the soil surface (Chen et al., 2007) improving infiltration (Adekalu et al., 2007), and soil water retention (Balwinder et al., 2011) and also led to improvements in crop yields in arid and semi-arid environments (Olasantan, 1999). Therefore, application of paddy straw mulch might have helped better conservation of soil moisture, which increased the availability of nutrients. On the other hand, no mulch treatment showed more water use and less water use efficiency among all the mulch treatments. Moreover, direct exposure of soil to sunlight and lack of mulch cover over the soil in control plots accentuated evaporation of water and thus, enhanced water use and reduced water use efficiency.

Manifestation of above results might be due to zinc deficiency in plants reduced the ability to respond to water stress due to failure of osmotic adjustment in the plant body (Khan et al., 2004). The active involvement of water in biomass production was also reduced due to zinc deficiency in plants (Khan et al., 2004). This was evident in the present experiment where the plants devoid of zinc reduced biomass production and showed low water use efficiency. On the other hand, boron has an important role in phosphorous metabolism and it is also involved in water relations (Nason and Elroy, 1963). The movement of boron from vegetative tissues to the actively growing meristamatic plant tissues (head) will depend on the activity of xylem channel in the plant (Shelp et al., 1995) and (Anonymous, 2016). More transpiration in plants grown under unmulched plots hampers the continuity of water flow in xylem channel, there by reduced the availability of applied boron to the apical bud (head), which resulted in development of poor quality heads with low yield and low water use efficiency. In contrast, plants grown under mulching and adequate of availability of boron through foliar spray, with sufficient availability of moisture with applied mulches and reduced transpiration might imported the better xylem transportation of boron to the apical meristem resulted increase in head weight with maximum water use efficiency. The beneficial effects of paddy straw mulch and micronutrients particularly $\mathrm{Zn}$ along with $\mathrm{B}$ as discussed above might have reduced water use and increased the water use efficiency. The present results are in conformity with findings of Sarkar et al., (2007) in yellow sarason, Rana (2009) and Lopez-Urrea et al., (2009) in broccoli and Prasad et al., (2014) in French bean.

\section{References}

Saha, P., Chatterjee, R. and Das, N. R. 2010. Effect of foliar application of boron and molybdenum in sprouting Broccoli (Brassica oleracea var italic Plenck) under Terai region of West Bengal. Research Journal of Agricultural Sciences 1(4): 335-337.

Brahma, S., Phookan, D. B., Gautam, B. P. and Bora, D. K. 2002. Effect of nitrogen, phosphorous and potassium on growth and yield of broccoli (Brassica oleracea L. var. italica) cv.KTS-1. Vegetable Science 29(2): 154-156.

Torres, F. P. 1974. Evaluation of zinc status of several Ecuadorian soils. https://books.google.co.in/books?id. 
Pandhawa, K. S. and Bhai, A.S. 1976. Growth yield and quality of cauliflower as influenced by nitrogen, phosphorous and boron. Indian Journal of Horticulture 33(1): 83-91.

Vitkov, M. 1975. The effect of irrigation and fertilization of the removal of main nutrients by the bean crop and top growth. Pochvoznamic N. Pushkarov, Sofia, Bulgaria 10:49-55

Panse, A.K, Sukhatme, P.V. 1978.Stastical Methods for Agriculture Workers. ICAR. New Delhi. pp. 97-123.

Chen, S.Y., Zhang, X.Y., Pei, D., Sun, H.Y. and Chen, S.L.2007 Effects of straw mulching on soil temperature, evaporation and yield of winter wheat: field experiments on the North China Plain. Annals of Applied Biology, 150: 261-268.

Adekalu, K.O., Olorunfemi, I.A., Osunbitan, J.A.2007.Grass mulching effect on infiltration, surface runoff and soil loss of three agricultural soils in Nigeria. Bioresource Technology 98: 912-917.

Balwinder-Singh, Humphreys, E., Eberbach, P.L., Katupitiya, A. and Yadvinder-Singh. 2011. Growth, yield and water productivity of zero till wheat as affected by rice straw mulch and irrigation schedule. Field Crops Research 121: 209-225.

Olasantan, F.O. 1999. Effect of time of mulching on soil temperature and moisture regime and emergence, growth and yield of white yam in western Nigeria. Soil \& Tillage Research 50: 215 221.

Khan, H. R., McDonald, G. K and Rengel, Z.2004.Zinc fertilization and water stress affects plant water relations, stomatal conductance and osmotic adjustment in chickpea (Citer arientinum L.). Plant and Soil 267(1-2), 271-284.

Nason, A and Me Elroy, W. D. 1963. Mode of action of the essential mineral elements In: F.C. Stoward (Ed.). Plant Physiol. 111 Inorganic nutrition of plants. Academic Press, New York. Pp.465-508.

Shelp, B. J., Marentes, E., Kitheka, A. M. and Vivekanandan, P. 1995. Boron mobility in plants. Physiologia Plantarum 94(2):356-361

Anonymous. 2016.Boron Nutrition for Optimal Plant Growth. Agri sight.issue: 12-2016.

Rana, R. S.(2009). Response of irrigation on broccoli yield under low hills sub-tropical conditions of Himachal Pradesh. Crop Research (Hisar), 37(1/3), 152-153.

Sarkar, S., Biswas, M., Goswami, S. B. and Bandyopadhyay, P. K. 2010. Yield and water use efficiency of cauliflower under varying irrigation frequencies and water application methods in Lower Gangetic Plain of India. Agricultural water management 97(10):1655-1662.

López-Urrea, R., Montoro, A., López-Fuster, P and Fereres, E.2009. Evapotranspiration and responses to irrigation of broccoli. Agricultural water management 96(7):1155-1161.

Prasad, B. V. G., Chakravorty, S., Saren, B. K. and Panda, D. 2014. Effect of mulching on physiological growth determinants of productivity in French bean (Phaseolus vulgaris L.). Hort Flora Research Spectrum, 3(2):162-165.

Parthasarathi, V.A. 1986. Bose, T.K. and M.G. Som (Eds.). Vegetable Crops in India Naya Prokash, Distributor, Calcutta, India. French bean. pp. 497-510.

\section{How to cite this article:}

Prasad B.V.G. and Chakravorty, S. 2019. Performance of Mulches and Micronutrients on Water Use of Broccoli (Brassica oleracea L var. Italica Plenck). Int.J.Curr.Microbiol.App.Sci. 8(01): 102-108. doi: https://doi.org/10.20546/ijcmas.2019.801.013 